\title{
Newborn Girl with Rapidly Progressive Linearly Distributed Vesicles and Pustules
}

Colin M Kenny ${ }^{*}$, Deptmer M Ashely and Sunghun Cho

Department of Medicine, Tripler Army Medical Center, Honolulu, HI

Department of Dermatology, Tripler Army Medical Center, Honolulu, HI

"Corresponding author: Colin M Kenny, Department of Medicine, Tripler Army Medical Center, 1 Jarrett White Rd, Honolulu, HI 96859, E-mail: colin.kenny@gmail.com

Received date: Dec 18, 2018; Accepted date: Jan 07, 2019; Published date: Jan 14, 2019

Copyright: @2019 Colin Kenny, et al. This is an open-access article distributed under the terms of the Creative Commons Attribution License, which permits unrestricted use, distribution, and reproduction in any medium, provided the original author and source are credited.

\begin{abstract}
One-day old infant girl, born at term, presents with blanching erythematous macules, vesicles and pustules scattered on her upper and lower extremities. These lesions progressed rapidly and began to coalesce and take on a linear configuration, even appearing on her trunk and face. A punch biopsy revealed eosinophilic spongiosis. Based on skin findings and histopathology this girl was diagnosed with Incontinentia pigmenti (IP) in accordance with the IP Diagnostic Criteria 2013. IP is a rare, X linked dominant genetic syndrome occurring in 1 of 50,000 births. IP presents with characteristic cutaneous lesions and in varying frequency, extracutaneous manifestations. The cutaneous manifestations appear in four progressive stages. The first stage consists of multifocal and coalescing erythematous macules and patches with overlying tense vesicles on the patient's trunk and extremities. These lesions are distributed along Blashko lines. In stage two, the lesions become hyperkeratotic appearing similar to viral warts. The lesions in the third stage darken and become hyperpigmented. They may appear as swirled macules. In the final stage hypopigmentation of the lesion occurs. Cutaneous symptoms often do not require additional medical care. Extracutaneous findings include dental, neurologic, and ocular anomalies. Requiring increased frequency of follow up by specialists during early childhood.
\end{abstract}

Keywords: Incontinentia pigmenti (IP); Dermatopathology; Blochsulzberger syndrome; Ectodermal dysplasia, Newborn

\section{Introduction}

To follow is brief case history and discussion regarding a rare dermatologic condition, Incontinentia pigmenti (IP). Often suspected soon after birth and subsequently confirmed with dermatopathology, early accurate diagnosis and treatment is essential in affected individuals. While primarily a cutaneous phenotype, IP affects a multitude of organ systems. As such, treatment of this condition requires a broad range of specialists to include: dermatologist, pediatricians, ophthalmologist, neurologist, and even adult medicine specialist. While rare, this diagnosis is based primarily on unique cutaneous findings, described below.

\section{Case Report}

A one-day old infant girl, born at $38+6$ weeks, via an uneventful spontaneous vaginal delivery presents with blanching erythematous macules, vesicles and pustules scattered sparsely on her upper and lower Extremities. Her gestation was remarkable for uncomplicated pre-eclampsia. The girl's mother past medical history was notable for impaired vision in her right eye and a genetic dermatologic disorder diagnosed in childhood. The infant girl's immediate post-partum physical exam was significant for a few scattered pink macules, papules, vesicles, and pustules all overlying a faint erythematous base on the medial aspects of her bilateral upper and lower extremities. The remainder of her physical exam was significant for physiologic acrocyanosis of the newborn. Within 24 hours, many more papules, vesicles, and pustules began to appear on her arms and legs with a few scattered on her abdomen and chin (Figure 1). As the lesions on her extremities multiplied they began to coalesce and take on a more linear configuration. A $2 \mathrm{~mm}$ punch biopsy was performed on her right forearm and revealed eosinophilic spongiosis with numerous eosinophil exocytosis into the epidermis (Figure 2). The mother had a diagnosis of incontinentia pigmenti without overt skin findings when she presented to deliver the baby. On the day of her birth, erythema toxicum neonatorum was considered in the differential but as the papules and pustules became more linearly distributed over the following hours, incontinentia pigmenti was suspected clinically. Soon after the final diagnosis was suspected, a neurologist and an ophthalmologist examined the girl. No neurologic or ophthalmologic concerns were identified. The patient was discharged from the hospital in good health.

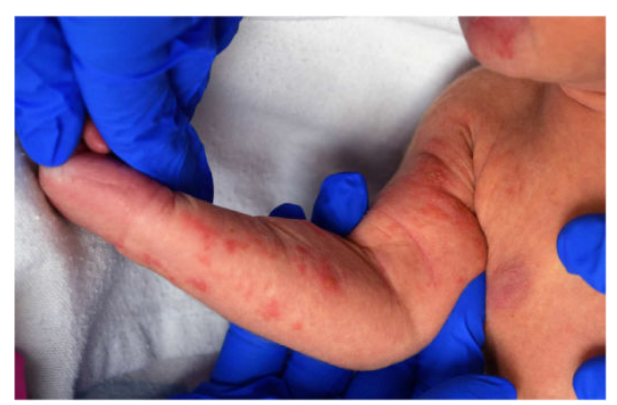

Figure 1: Skin Erythematous papules, vesicles, and pustules over an erythematous base on the patient's arm. Some of the lesions are coalescent; others are arranged linearly. 


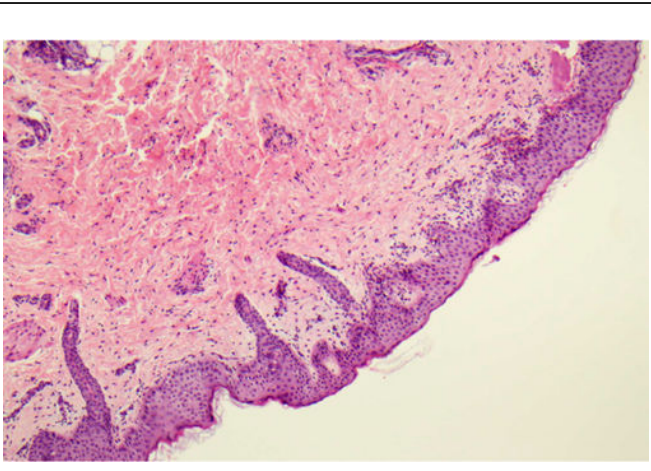

Figure 2: Histopathology Eosinophilic spongiosis with numerous eosinophils showing exocytosis into the epidermis. Apoptotic Keratinocytes and dyskeratosis are present.

\section{Discussion}

The girl was diagnosed with Incontinentia pigmenti as her skin eruptions and histopathologic findings met both the major and one minor clinical criterion outlined in Incontinentia Pigmenti Diagnostic Criteria 2013 update [1].

Incontinentia pigmenti diagnostic criteria were first formalized in 1993 but revised and updated in 2013 to reflect the addition of genetic testing to the diagnostic criteria. To establish the diagnosis a patient must display characteristic progressive cutaneous symptoms. This is the only major clinical diagnostic criteria and a requirement for the diagnosis of IP. This often requires a high index of clinical suspicion when examining a newborn who maybe in the early vesicular stage or later in life when the cutaneous lesions become less pronounced [1].

Minor clinical criteria which support the diagnosis of IP includes:

- Typical histopathologic finding on skin biopsy

- Alopecia or hair loss/growth irregularities

- Central nervous system abnormalities

- Nail dystrophy

- Dental malformations

- History of multiple confirmed male miscarriages

- Palate anomalies

- Breast abnormalities [1]

The current diagnostic clinical criteria require typical skin findings and one or more minor criteria. The 2013 diagnostic update also includes IкBKG/NEMO mutations, discoverable through genetic testing, as a single criterion usable for diagnosis [1]. If IP is suspected in a male patient, the individual should undergo testing for somatic mosaicism and/or Klinefelter syndrome [2].

Incontinentia pigmenti (IP), also known as Bloch-Sulzberger syndrome, is an X-linked dominant genetic syndrome with characteristic cutaneous lesions and in varying frequency extracutaneous manifestations. IP is cause by loss of function mutations in regulatory proteins, inhibitor of kappa polypeptide gene enhancer in B-cells/nuclear factor kappa B essential modulator (IкBKG), formerly NEMO. These regulatory proteins are involved in many cellular processes, including apoptosis and inflammation modulation. X-linked is the only identified mode of inheritance. However, approximately 65 percent of cases occur as de novo mutations without identifiable genotype-phenotype correlation. This syndrome occurs in approximately $1: 50,000$ births and is predominantly observed in females which account for 97 percent of cases [3]. Often male fetuses with this mutation die in utero. Several case reports have described males born with this condition; however, these males either had somatic mosaicism or Klinefelter karyotype [2]. As this syndrome has cutaneous, hair, nail abnormalities, and central nervous system involvement, is classified as a form of ectodermal dysplasia [3].

The majority of initial cutaneous manifestation of IP is often noticed at birth or shortly thereafter, with the remainder within the first four months of life. Four characteristic progressive stages of are classically described; however the fourth stage is often absent. This first stage, as described in this case, consists of multifocal and coalescing erythematous macules and patches with overlying tense vesicles on the trunk and extremities. Pustules may or may not be present. These lesions must be distributed along Blashko lines, a developmental growth pattern that occurs during epidermal migration. CBC differentiated during this stage will often demonstrate eosinophilia. This vesicular stage may persist for severalmonths, and will often reoccur during general febrile illnesses early in life [4,5]. Stage two, termedverrucous, follows the resolution of stage one. The lesions become hyperkeratotic with an occasional overlying crust, appearing very similar to viral warts. These lesions remain localized to Blaschko lines. These lines are often whorled on the trunk and linear on the extremities, as in our patient. Stage three, apply named hyperpigmented stage, occurs within 6 to 12 months of stage 1. In this stage, the lesions observed in the first two stages darken to brown or grey-brown and may appear as swirled macules. The hyperpigmented macules and patches generally persist through adolescence but may remain even into adulthood. The hyperpigmentation often fades gradually until it resembles surrounding unaffected skin and becomes unidentifiable. As such the fourth stage, hypopigmented stage, often does not occur as was the case with the patient's mother. When this stage is observed, the hyperpigmented macules and patches fade until they become hypopigmented and take on a slightly atrophic appearance [4].

Extracutaneous findings in IP appear in varying incidence. A wide range of dental abnormalities are observed in the many IP cases. Classically, pegged or conical teeth are associated with IP; however, delayed dentition, anodontia, and hypodontia have also been described. Approximately 50 percent of IP patients will suffer from some form of alopecia or other hair related anomalies on their face and scalp. Neurologic symptoms with central nervous system abnormalities, observable on MRI, affect approximately 30 percent of IP patients. These symptoms range from lethargy and poor feeding to seizures, spastic paralysis, mental retardation, and developmental delays. If present, these symptoms manifest early in life [4]. In addition, proliferative retinopathy and peripheral avascularityare known to occur in greater frequency, in up to $1 / 3$ of IP patients, leading to vision impairment or even total blindness [6]. Manifesting later in life, even into early adulthood, 10 percent of IP patients will experience nail dystrophy manifested as onychogryphosis, or missing nails. A smaller subset of patients will have radiographic evidence of distal phalange osteolytic lesions [4]. 
Citation: Colin M Kenny, Deptmer M Ashely, Sunghun Cho (2019) Newborn Girl with Rapidly Progressive Linearly Distributed Vesicles and Pustules. Dermatol Case Rep 4: 146.

Page 3 of 3

Treatment of cutaneous symptoms generally does not require specialty care apart from topical emollients. Occasionally, if warranted, topical or oral antibiotics for resultant skin and soft tissue infection occurring during stage one and two [4]. Management of extra cutaneous symptoms vary depending on the individual patient symptoms. A paediatric dental consultation is recommended around age one or at the time of first teeth eruption [4]. Due to the high percentage of IP patients with ophthalmologic complaints, patients should receive monthly eye exams for the first four months of life, followed by an exam every three months until age one, then six months until age three. If exams remain benign, yearly exams often suffice beyond age three [6]. Many pediatric neurologist also wish to evaluate IP patients every three months during the infant' first year [4].

\section{References}

1. Minić S, Trpinac D, Obradović M(2013) Incontinentia pigmenti diagnostic criteria update. Clin Genet 85: 536-542.
2. Buinauskaite E, Buinauskiene J, Kucinskiene V, Strazdiene D, Valiukeviciene S (2010) Incontinentia pigmenti in a male infant with Klinefelter syndrome: a case report and review of the literature. Pediatric Derma 27: 492-495.

3. Narayanan MJ, Rangasamy S, Narayanan V (2015) Incontinentia pigmenti (Bloch-Sulzberger syndrome). Handbook of Clinical Neurology 132: 271-280.

4. Bodemer C (2013) Incontinentia pigmenti and hypomelanosis of Ito. Handbook of Clinical Neurology 111: 341-347.

5. Van Leeuwen RL, Wintzen M, van Praag MC (2000) Incontinentia pigmenti: an extensive second episode of a "first-stage" vesicobullous eruption. Pediatric Derma 17: 70.

6. Swinney CC, Han DP, Karth PA (2015) Incontinentia pigmenti: A comprehensive review and update. Ophthalmic Surgery Lasers Imaging Retina 46: 650-657. 